\title{
Consecutive severe immune-related adverse events after PD-1 inhibitor induction and surgery in locally advanced non-small cell lung cancer: a case report
}

\author{
Hang Zhao ${ }^{1,2 \#}$, Junwei Ning ${ }^{1 \#}$, Yu Gu ${ }^{3 \#}$, Xiaocheng Zhang ${ }^{1,4}$, Wen Yu $^{5}$, Tianxiang Chen ${ }^{1}$, Qingquan Luo ${ }^{1}$ \\ ${ }^{1}$ Shanghai Lung Cancer Center, Shanghai Chest Hospital, Shanghai Jiao Tong University, Shanghai, China; ${ }^{2}$ Thoracic Surgery Department, \\ Dongyang People's Hospital, Dongyang, China; ${ }^{3}$ Department of Radiation Oncology, Fudan University Shanghai Cancer Center, Shanghai, China; \\ ${ }^{4}$ Department of Pulmonary Medicine, Ruian People's Hospital, Wenzhou, China; ${ }^{5}$ Department of Radiation Oncology, Shanghai Chest Hospital, \\ Shanghai Jiao Tong University, Shanghai, China \\ \#These authors equally contributed to this work. \\ Correspondence to: Qingquan Luo; Tianxiang Chen. Shanghai Lung Cancer Center, Shanghai Chest Hospital, Shanghai Jiao Tong University, 241 \\ Huai Hai Road, Shanghai 200030, China. Email: luoqingquan@hotmail.com; txchen@shsmu.edu.cn.
}

\begin{abstract}
Neoadjuvant PD-(L)1 inhibitors may be promising for locally advanced non-small cell lung cancer (NSCLC) with potential pathological and survival benefits. But severe immune-related adverse events (irAEs) may cause fatal consequences which require early identification and timely intervention. The basis for most of these adverse events is a reactive hyperactivated T-cell response to normal tissues that results in the production of high levels of CD4 T-helper cell cytokines or increased migration of cytolytic CD8 T cells in normal tissues. It is recommended that all patients receiving PD-(L)1 inhibitors routinely have thyroid function studies, complete blood counts, and liver function and metabolic panels at each treatment and at intervals of 6 to 12 weeks for the first 6 months after finishing treatment. Herein, we report a rare case who had two grade 3-4 irAEs consecutively occurring after PD-1 induction therapy and surgery. A 59-year-old man with stage IIIA squamous cell lung cancer receiving 3 cycles of neoadjuvant nab-paclitaxel, carboplatin, and nivolumab was reevaluated for resectability. The patient experienced acute serum transaminase elevation right after induction therapy. Seven days after surgery he had severe pneumonia. These two serious complications were both eventually relieved by a month long treatment of corticosteroids but not regular medicine which verified the diagnosis of irAEs. Although results of clinical trials of neoadjuvant immunotherapy are worth expecting, our case calls attention to careful surveillance and timely management of irAEs during the perioperative use of PD-(L)1 inhibitors. We also further discuss the standard use of corticosteroids for irAEs based on a review of literature.
\end{abstract}

Keywords: Hepatitis; pneumonia; immune-related adverse events (irAEs); non-small cell lung cancer (NSCLC); case report

Submitted Jun 22, 2021. Accepted for publication Aug 16, 2021.

doi: $10.21037 /$ tlcr-21-603

View this article at: https://dx.doi.org/10.21037/tlcr-21-603

\section{Introduction}

In recent years, immune checkpoint inhibitors (ICIs) targeting PD-1/PD-L1 for advanced non-small cell lung cancer (NSCLC) have shown promising results in clinical trials, and are recognized as the standard treatment (1-3). Recent clinical studies like Checkmate-816 also proved that

\footnotetext{
$\wedge$ ORCID: 0000-0002-0032-0026.
} 

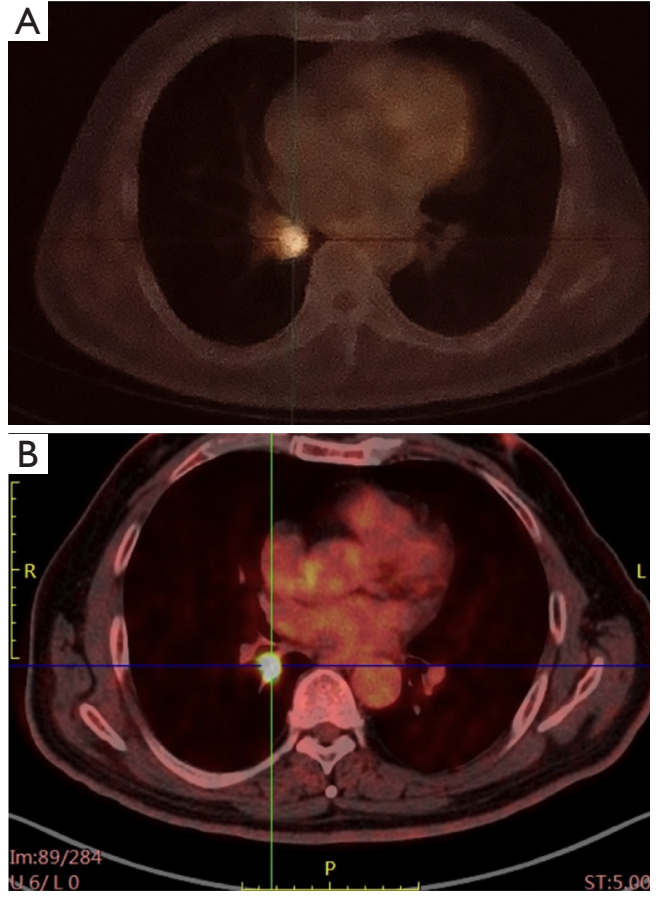

Figure 1 PET-CT images (A) before induction; (B) before surgery. (A) The tumor was observed at the bronchi in the dorsal segment of the lower lobe of the right lung when admitted. The diameter was $2.5 \mathrm{~cm}$, SUVmax 11.2 (standard uptake value, maximum), with lymph nodes metastasis in the group 4R and 7; (B) the tumor 10 days before surgery. It was considered that the malignant lesion still had high metabolic activity. The size was about $1.5 \mathrm{~cm} \times$ $1.12 \mathrm{~cm}$, and the enhanced SUVmax was 11.8 .

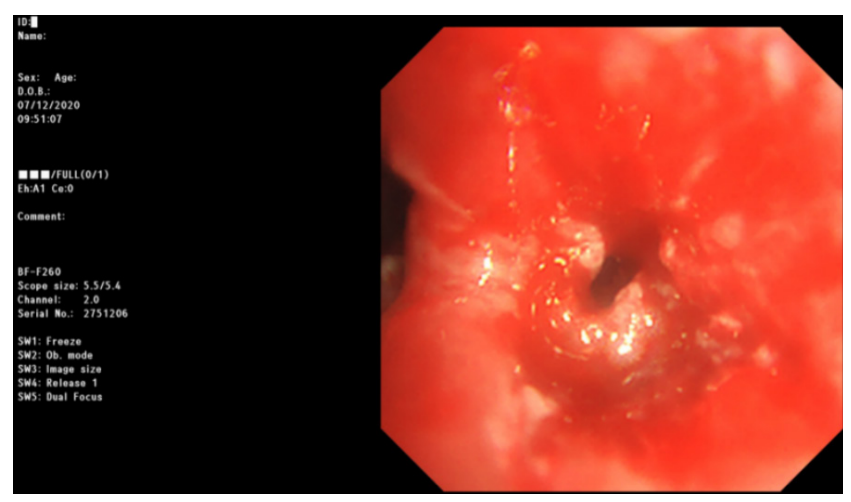

Figure 2 Endoscopic view of right lower bronchus after transbronchial biopsy. The right middle bronchial mucosa was hyperemic, swollen, and red in fluorescence, and the right lower bronchus was completely blocked. neoadjuvant immunotherapy for locally advanced NSCLC achieve satisfactory rate of major pathologic response (MPR) and pathologic complete response (pCR) (4-7).

Among the immune-related adverse events (irAEs) of PD-(L)1 inhibitor, hepatitis and pneumonia are considered to be occasional and mild, mostly graded 1-2 (8). However, in reality severe irAEs after immune neoadjuvant therapy not only caused delays or difficulty of surgery, but also increased rate of fatal postoperative complications. Therefore, early identification and timely intervention for irAEs is clinically crucial. In this case report, we present the detailed treatment and outcome of a patient with locally advanced NSCLC who manifested ICI-related hepatitis after induction treatment and postoperative life-threatening ICI-related pneumonia. We present the following article in accordance with the CARE reporting checklist (available at https://dx.doi.org/10.21037/tlcr-21-603).

\section{Case presentation}

A 59-year-old Chinese man with body mass index of $27.4 \mathrm{~kg} / \mathrm{m}^{2}$ who had a 30 pack-year smoking history was admitted to our center due to a pulmonary right lower lobe tumor close to hilum discovered by chest scan without obvious symptoms. Positron emission tomography/ computed tomography (PET-CT) in November 2020 showed a mass, which was $25 \mathrm{~mm}$ in diameter and had a maximum standard uptake value (SUVmax) of 11.2, with suspected station $4 \mathrm{R}$ and 7 lymph node metastasis in the right hilar (Figure 1). Transbronchial biopsy confirmed squamous cell carcinoma and complete obstruction in the right lower bronchus (Figure 2). He had well-controlled depression by regular medication (citalopram hydrobromide tablet), uncontrolled slight blood glucose elevation $(6.2 \mathrm{mmol} / \mathrm{L})$, and controlled hypertension for over 20 years by regular medication without other cardiovascular disease or any other comorbidity.

Before any treatment, his liver function tests including alanine aminotransferase (ALT) and aspartate aminotransferase (AST) were normal, and laboratory tests ruled out hepatitis $\mathrm{A} / \mathrm{C}$ virus infection, but indicated that he was an asymptomatic hepatitis B virus carrier. After multidisciplinary team discussion, he was considered inoperable with multiple stage IIIA (N2) disease and an Eastern Cooperative Oncology Group (ECOG) performance status score of 1 . Next-generation sequencing (NGS) revealed no classical driver mutations within the tumor cell genome, but the expression of PD-L1 in 
Table 1 Cycles of chemoimmunotherapy

\begin{tabular}{|c|c|c|c|c|}
\hline Cycle & Dose & $\mathrm{AE}$ & Treatment & Response \\
\hline 3 & $\begin{array}{l}\text { Nab-paclitaxel } 475 \text { mg, carboplatin } \\
520 \text { mg, nivolumab } 360 \mathrm{mg}\end{array}$ & $\begin{array}{l}\text { Immune-related hepatitis ( } 22 \text { days after treatment); } \\
\text { immune-related pneumonia ( } 64 \text { days after treatment) }\end{array}$ & - & PR \\
\hline
\end{tabular}

$\mathrm{AE}$, adverse event; CSF, colony-stimulating factor; PR, partial response.

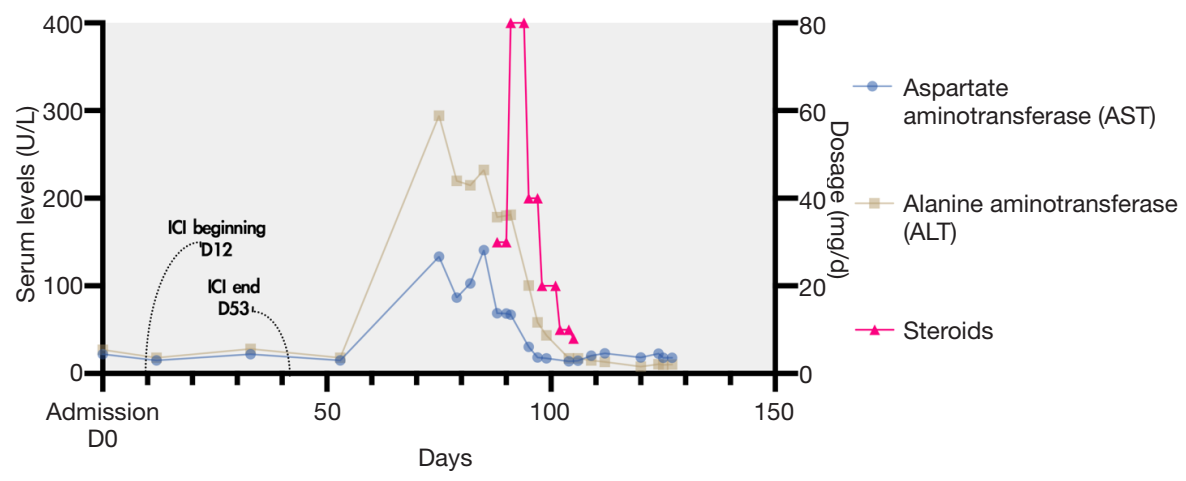

Figure 3 Liver parameters and corticosteroids use. Say the admission day of this patient was day 0 (D0, etc.), the ICI induction therapy began on D12, and ended on D53. On D75, it was found that elevated serum level of ALT at 294 U/L and AST at 133 U/L. Intravenous methylprednisolone was started with $30 \mathrm{mg}$ per day $(30 \mathrm{mg} / \mathrm{d})$ on D88. And on D91, it reached a maximum dosage of $80 \mathrm{mg} / \mathrm{d}$, then it gradually reduced to $10 \mathrm{mg} / \mathrm{d}$ every 4 days until oral methylprednisolone was prescribed at $8 \mathrm{mg} / \mathrm{d}$ on D105. Corticosteroids were stopped 4 days before surgery.

tumor tissues was over $10 \%$. Given the NGS results and the surgically removable tumor type, targeted therapy was dismissed. Because the patient and his family had a strong desire for surgery, the medical team decided that a tentative induction therapy would be first given to see if there was chance to downstage the patient for surgery. $\mathrm{He}$ underwent 3 cycles of neoadjuvant chemoimmunotherapy starting with nab-paclitaxel $(475 \mathrm{mg})$, carboplatin $(580 \mathrm{mg})$, and nivolumab $(360 \mathrm{mg})$ every 3 weeks, and a partial response (shrinkage from 28 to $19 \mathrm{~mm}$ in diameter) was achieved (Table 1). Meanwhile, damage of liver function was developed 22 days after the last cycle with elevated serum level of ALT at 294 U/L and AST at 133 U/L.

Since patient had hepatitis B, a course of therapy with magnesium isoglycyrrhizinate and reduced glutathione was first used to protect liver function which was regular combination to reduce liver transaminases in China. However, both ALT and AST levels remained high and other laboratory parameters were within normal ranges, immune-related hepatitis might be the possible cause which could not be relieved without corticosteroids. We added methylprednisolone starting from $30 \mathrm{mg}$ to maximum dose at $80 \mathrm{mg}$ to therapy 13 days after transaminases elevation. ALT and AST soon declined to normal within 10 days. Then the dose of methylprednisolone was gradually reduced every 4 days to the minimum oral dose at $8 \mathrm{mg}$ within 10 days. For the preparation of surgery, corticosteroids were suspended 4 days before operation (Figure 3).

PET-CT (Figure 1) after liver function being normal showed that the SUVmax declined to 11.8 without any suspected metastasis of $\mathrm{N} 2$ lymph nodes, so the patient was clinically restaged as cT2 $\mathrm{NxM} 0$, stage IBIIIA, and the tumor was reconsidered to be resectable. The patient did not have a cough, sputum, chest pain, dyspnea, or other symptoms, and prophylactic antibiotics were prescribed. Sixty days after neoadjuvant therapy, the patient uneventfully received open surgery of right middle and lower bilobectomy with blood loss about 

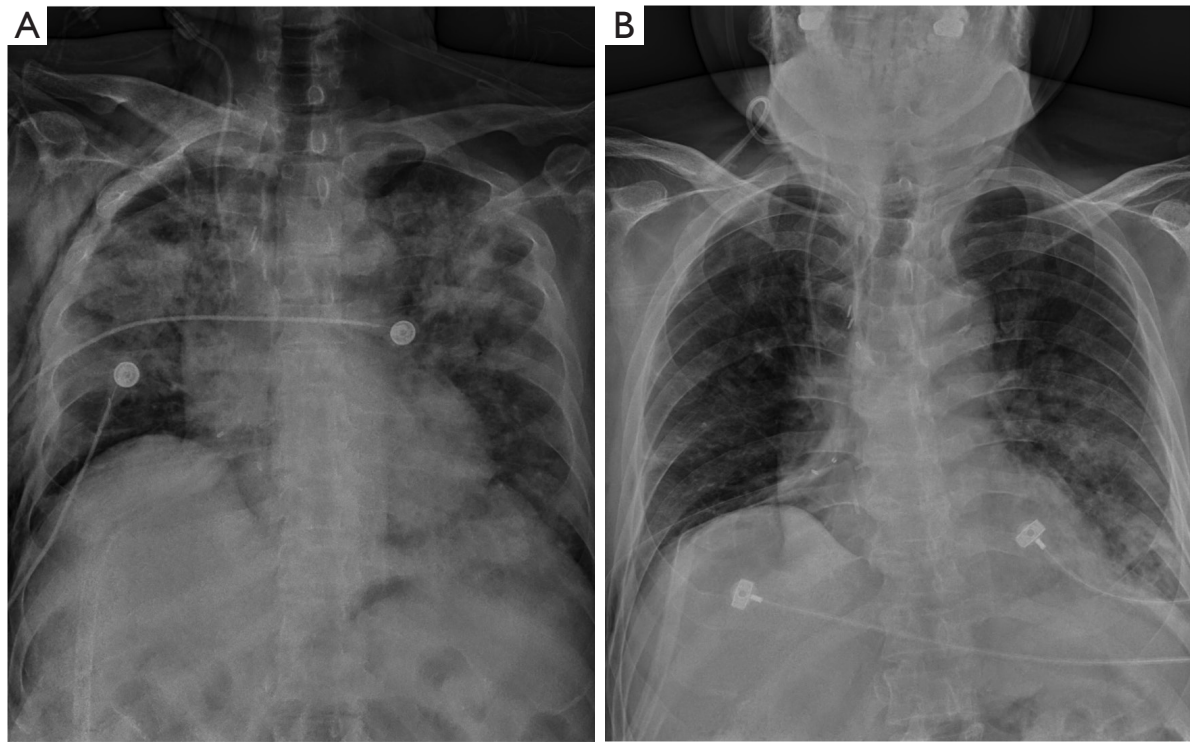

Figure 4 Bedside chest X-ray images (A) after surgery; (B) after steroids. (A) The bedside chest X-ray image 9 days after surgery showed severe bilateral pneumonia; (B) the bedside chest X-ray image cleared 4 days after corticosteroids treatment.

$100 \mathrm{~mL}$. On the 5th postoperative day (POD), his highest body temperature was $38.1^{\circ} \mathrm{C}$, and CT showed scattered inflammatory lesions in both lungs that indicated possible postoperative pulmonary infection. His chest tube was unobstructed without air leak, and he was treated with empirical antibiotic treatment of imipenem/cilastatin. On the 7th POD, hypoxia was observed with finger pulse oxygen about $75 \%$ saturation when the patient was inhaling oxygen. After oxygen inhalation by face mask delivering oxygen concentration of $40 \%$ flowing at $5 \mathrm{~L} / \mathrm{min}$, his oxygenation was back to $95-96 \%$. The D-dimer level was tested to be $1.85 \mathrm{mg} / \mathrm{L}$, postoperative deep vein thrombosis could not be easily ruled out, therefore, low-molecular-weight heparin was administered for anticoagulation although without strong evidence. On the 8th POD, the patient was transferred to the intensive care unit for bi-level positive airway pressure (BiPAP) therapy because of severe hypoxia with oxygen saturation of $60-70 \%$ with face-mask oxygen inhalation. A bedside $\mathrm{X}$-ray suggested bilateral severe pulmonary infection (Figure 4). The patient was then diagnosed with immune-related pneumonia given the history of preoperative immunerelated liver damage, we administered both intravenous methylprednisolone $(1 \mathrm{mg} / \mathrm{kg})$ along with gamma globulin $(20 \mathrm{~g})$ to bring down the immune related complications. In addition, oxygen therapy by BiPAP and nutritional support were also strengthened. The hypoxia symptom was relieved very soon after corticosteroids treatment, 13th POD chest $\mathrm{X}$-ray indicated greatly improved pulmonary condition (Figure 4), gamma globulin treatment was stopped. The patient were sent back to normal surgery ward and discharged on 25 th POD. After 17 days postoperative course of corticosteroids, he continued oral prednisone acetate tablets for 28 days from the day of discharge.

All procedures performed in studies involving human participants were in accordance with the ethical standards of the institutional and/or national research committee(s) and with the Helsinki Declaration (as revised in 2013). Written informed consent was obtained from the patient for publication of this case report and accompanying images. A copy of the written consent is available for review by the editorial office of this journal.

\section{Discussion}

In the last decade, significant advances have been achieved in the treatment of NSCLC $(9,10)$. Currently, a number of phase III clinical studies of neoadjuvant immunotherapy including CheckMate 816, Keynote-671, and IMPOWER030 among others are under way. Although the potential benefit of neoadjuvant immunotherapy was seen in the early phase I/II clinical studies, some immunotherapy related concerns were also noticed (4,11-13). For example, 


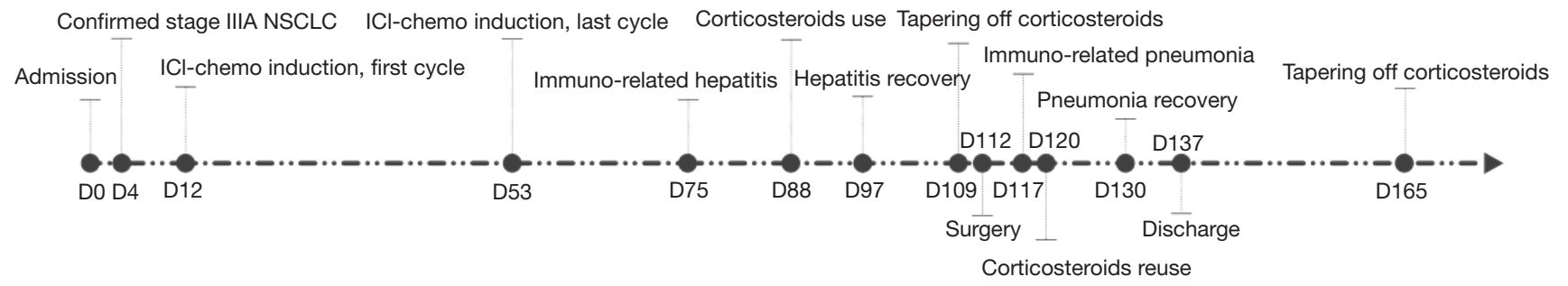

Figure 5 Treatment timeline of our case. Confirmed NSCLC refers to historically confirmed squamous cell lung cancer. The day of admission was set for day 0 (D0). Corticosteroids include methylprednisolone during hospital stay and prednisone after discharge.

of 90 patients with resectable stage IB-IIIA NSCLC in the LCMC3 study, 30 patients $(30 \%)$ had irAEs (1 case grade $\geq 3$ ) after atezolizumab was administered for 2 cycles preoperatively (14). The NADIM study reported that the incidence of postoperative complications was $29 \%(12 / 41)$ after 3 cycles of preoperative chemotherapy with nivolumab plus carboplatin and paclitaxel (4). Another study reported an approximately $30 \%(6 / 20)$ incidence of atrial arrhythmia, a $5 \%$ incidence of myocardial infarction, a $5 \%$ incidence of pulmonary infection, a $5 \%$ incidence of pulmonary embolism, and a 5\% incidence of empyema after 2 cycles of nivolumab alone for NSCLC (15). Therefore, it can be seen from the published data of various studies that some patients suffer from adverse events or other reasons which may lead to a delay in surgery, reduced surgical safety, higher rate of conversion, prolonged hospital stay, increased economic burden, or even perioperative morbidity and mortality.

Our patient developed immune related hepatitis right after induction which could not be relieved by hepatoprotective medicine (Figure 5), and the liver enzyme levels returned back to normal after timely steroids therapy. Unfortunately, he developed immune-related pneumonia one week after surgery, which still could not be improved by antibiotics alone, the patient was sent to intensive care unit to get support with BiPAP therapy and treated with high-dose corticosteroids, immunoglobulin along with antibiotics. With gradually reduced steroid doses and a series of relatively longterm anti-inflammatory maintenance therapies, his condition improved with satisfactory outcomes.

irAEs are usually organ specific. Dermatologic, gastrointestinal, endocrine, respiratory, and hepatic adverse reactions are most frequently reported in clinical trials (16). In KEYNOTE-001 (17), patients with a history of chest radiotherapy were more likely to have treatment-related lung injury after pembrolizumab than those without previous chest radiotherapy (13\% versus $1 \%)$. For grade 3-4 immune-related pneumonia, the American Society of Clinical Oncology/National Comprehensive Cancer Network (ASCO/NCCN) recommends $1-2 \mathrm{mg} / \mathrm{kg} / \mathrm{d}$ methylprednisolone or prednisolone (18). The time of tapering should be more than 6 weeks. The European Society for Medical Oncology (ESMO) recommends 2-4 mg/ $\mathrm{kg} / \mathrm{d}$ methylprednisolone or prednisolone, and the tapering time is more than 8 weeks (19). The ASCO/ NCCN guideline suggests that when pneumonia reverts to grade 1, steroids can be reduced. ESMO indicates that steroids cannot be reduced until lung inflammation has recovered to basal levels. Due to the lack of a direct comparison of these two therapeutic strategies, both can be taken into practice. There is far from consensus as to what subsequent treatment options should be chosen for patients with poor response to corticosteroid therapy. Both the ASCO/NCCN and ESMO guidelines list treatment options such as infliximab, mycophenolate mofetil, and gamma globulin. However, corticosteroids may reduce the efficacy of PD-(L)1 inhibitors, which may negatively affect the efficacy of ICIs. Data supporting this view come from patients with NSCLC and melanoma, who are most often treated with ICIs $(20,21)$. In these patients, steroids are associated with poorer outcomes and reduced progressionfree survival, so treatments other than corticosteroids are required. According to a review, the overall incidence of severe or life-threatening irAEs (grade $\geq 3$ ) ranged from $10 \%$ to $15 \%$ for patients with PD-1 inhibitors, and toxicities appear to be dose-independent. While rare, ICIrelated pneumonitis is considered one of the more serious irAEs (22-24). The incidence of pneumonitis in melanoma patients receiving anti-PD-1 monotherapy and combination therapy is $3.8 \%$ and $9.6 \%$, respectively (25). And the occurrence of grade 3 hepatitis after ICIs is about $2 \%$ in NADIM study and other trials $(4,26)$.

In this case, 2 irAEs (one grade 4) affecting different organs occurred during the perioperative period, which is rare. This also provided us with lessons and further thinking: 
if immune-related hepatitis occurs after induction therapy, would full course of steroids therapy prevent further postoperative irAEs, despite a delay in surgery? Because the long-term steroids therapy might highly increase the risk of bronchial anastomotic leakage, this patient did not further receive sleeve resection to achieve $\mathrm{R} 0$ resection after frozen pathology indicated positive bronchial margin. Lifethreatening immune-related pneumonia occurred after surgery which also required long-term high-dose steroids therapy. He was lucky to avoid bronchial anastomotic leakage perioperatively, however it was challenging to strategize adjuvant therapy. Whether or not and when to add radiotherapy to cover the positive margin and mediastinal lymph nodes were not easy to decide. Adjuvant immunotherapy would not be suggested since two irAEs occurred in this patient. Though irAEs could be rarely fatal, but the promising survival benefits for lung cancer should not be overlooked. The keys to successfully managing ICIs toxicity are early diagnosis, high suspicion, good patientprovider communication, and rapid and aggressive use of corticosteroids and other immunosuppressants. To date, there are no effective biomarkers to predict the toxicity of immunotherapy and this is an active area of research.

In conclusion, although neoadjuvant immunotherapy has potential pathological and survival benefits for patients with locally advanced NSCLC, it is difficult to avoid perioperative occurrence of irAEs, which can be fatal and disastrous. Therefore, the proper and timely management of irAEs needs to be further carefully standardized, and relevant therapeutic principles still need to be further explored.

\section{Acknowledgments}

Funding: This article was supported by the National Natural Science Foundation of China (Grant No. 81972176, 81601995), Shanghai Science and Technology Committee (18411966100), Shanghai Talent Development Fund (2019073), Shanghai Natural Science Foundation (Grant No. 18ZR1435100), Shanghai Rising Stars of Medical Talent, Youth Development Program (Specialist Program), Morningstar award program for outstanding young scholars of Shanghai Jiao Tong University.

\section{Footnote}

Reporting Checklist: The authors have completed the CARE reporting checklist. Available at https://dx.doi. org/10.21037/tlcr-21-603
Conflicts of Interest: All authors have completed the ICMJE uniform disclosure form (available at https://dx.doi. org/10.21037/tlcr-21-603). The authors have no conflicts of interest to declare.

Ethical Statement: The authors are accountable for all aspects of the work in ensuring that questions related to the accuracy or integrity of any part of the work are appropriately investigated and resolved. All procedures performed in studies involving human participants were in accordance with the ethical standards of the institutional and/or national research committee(s) and with the Helsinki Declaration (as revised in 2013). Written informed consent was obtained from the patient for publication of this case report and accompanying images. A copy of the written consent is available for review by the editorial office of this journal.

Open Access Statement: This is an Open Access article distributed in accordance with the Creative Commons Attribution-NonCommercial-NoDerivs 4.0 International License (CC BY-NC-ND 4.0), which permits the noncommercial replication and distribution of the article with the strict proviso that no changes or edits are made and the original work is properly cited (including links to both the formal publication through the relevant DOI and the license). See: https://creativecommons.org/licenses/by-nc-nd/4.0/.

\section{References}

1. Borghaei H, Paz-Ares L, Horn L, et al. Nivolumab versus Docetaxel in Advanced Nonsquamous Non-Small-Cell Lung Cancer. N Engl J Med 2015;373:1627-39.

2. Rittmeyer A, Barlesi F, Waterkamp D, et al. Atezolizumab versus docetaxel in patients with previously treated non-small-cell lung cancer (OAK): a phase 3, openlabel, multicentre randomised controlled trial. Lancet 2017;389:255-65.

3. Gettinger S, Rizvi NA, Chow LQ, et al. Nivolumab Monotherapy for First-Line Treatment of Advanced NonSmall-Cell Lung Cancer. J Clin Oncol 2016;34:2980-7.

4. Provencio M, Nadal E, Insa A, et al. Neoadjuvant chemotherapy and nivolumab in resectable non-small-cell lung cancer (NADIM): an open-label, multicentre, singlearm, phase 2 trial. Lancet Oncol 2020;21:1413-22.

5. Spicer J, Wang CL, Tanaka F, et al. Surgical outcomes from the phase 3 CheckMate 816 trial: Nivolumab (NIVO) + platinum-doublet chemotherapy (chemo) vs chemo 
alone as neoadjuvant treatment for patients with resectable non-small cell lung cancer (NSCLC) [EB/OL]. ASCO 2021:abstract 8503 .

6. Chen T, Ning J, Campisi A, et al. Neoadjuvant PD-1 inhibitors and chemotherapy for locally advanced NSCLC: A retrospective study. Ann Thorac Surg 2021. [Epub ahead of print]. doi: 10.1016/j.athoracsur.2021.03.041.

7. Jiang L, Huang J, Jiang S, et al. The surgical perspective in neoadjuvant immunotherapy for resectable nonsmall cell lung cancer. Cancer Immunol Immunother 2021;70:2313-21.

8. Ascierto PA, Del Vecchio M, Mandalá M, et al. Adjuvant nivolumab versus ipilimumab in resected stage IIIB-C and stage IV melanoma (CheckMate 238): 4-year results from a multicentre, double-blind, randomised, controlled, phase 3 trial. Lancet Oncol 2020;21:1465-77.

9. Duma N, Santana-Davila R, Molina JR. Non-Small Cell Lung Cancer: Epidemiology, Screening, Diagnosis, and Treatment. Mayo Clin Proc 2019;94:1623-40.

10. Chen T, Luo J, Gu H, et al. Impact of Solid Minor Histologic Subtype in Postsurgical Prognosis of Stage I Lung Adenocarcinoma. Ann Thorac Surg 2018;105:302-8.

11. Lee J, Chaft J, Nicholas A, et al. P2.04-88 Surgical outcomes of a multicenter phase II trial of neoadjuvant atezolizumab in resectable stages Ib-IIIb NSCLC: Update on LCMC3 clinical trial. J Thorac Oncol 2019;14:S744.

12. Sepesi B, Cascone T, William W, et al. OA13.06 Surgical outcomes following neoadjuvant nivolumab or nivolumab plus ipilimumab in nonsmall cell lung cancer - NEOSTAR study. J Thorac Oncol 2019;14:S241-S242.

13. Gao S, Li N, Gao S, et al. Neoadjuvant PD-1 inhibitor (Sintilimab) in NSCLC. J Thorac Oncol 2020;15:816-26.

14. Surgical and Clinical Outcomes With Neoadjuvant Atezolizumab in Resectable Stage IB-IIIB NSCLC: LCMC3 Trial Primary Analysis. Presented at: 2020 World Conference on Lung Cancer Singapore. January 28-31, 2021. PS01.05.

15. Bott MJ, Yang SC, Park BJ, et al. Initial results of pulmonary resection after neoadjuvant nivolumab in patients with resectable non-small cell lung cancer. J Thorac Cardiovasc Surg 2019;158:269-76.

16. Darnell EP, Mooradian MJ, Baruch EN, et al. ImmuneRelated Adverse Events (irAEs): Diagnosis, Management, and Clinical Pearls. Curr Oncol Rep 2020;22:39.

17. Shaverdian N, Lisberg AE, Bornazyan K, et al. Previous radiotherapy and the clinical activity and toxicity of pembrolizumab in the treatment of non-small-cell lung cancer: a secondary analysis of the KEYNOTE-001 phase 1 trial. Lancet Oncol 2017;18:895-903.

18. Brahmer JR, Lacchetti C, Schneider BJ, et al. Management of Immune-Related Adverse Events in Patients Treated With Immune Checkpoint Inhibitor Therapy: American Society of Clinical Oncology Clinical Practice Guideline. J Clin Oncol 2018;36:1714-68.

19. Haanen JBAG, Carbonnel F, Robert C, et al. Management of toxicities from immunotherapy: ESMO Clinical Practice Guidelines for diagnosis, treatment and followup. Ann Oncol 2017;28:iv119-42.

20. Arbour KC, Mezquita L, Long N, et al. Impact of Baseline Steroids on Efficacy of Programmed Cell Death-1 and Programmed Death-Ligand 1 Blockade in Patients With Non-Small-Cell Lung Cancer. J Clin Oncol 2018;36:2872-8.

21. Faje AT, Lawrence D, Flaherty K, et al. High-dose glucocorticoids for the treatment of ipilimumab-induced hypophysitis is associated with reduced survival in patients with melanoma. Cancer 2018;124:3706-14.

22. Spain L, Diem S, Larkin J. Management of toxicities of immune checkpoint inhibitors. Cancer Treat Rev 2016;44:51-60.

23. Kumar V, Chaudhary N, Garg M, et al. Current Diagnosis and Management of Immune Related Adverse Events (irAEs) Induced by Immune Checkpoint Inhibitor Therapy. Front Pharmacol 2017;8:49.

24. Widmann G, Nguyen VA, Plaickner J, et al. Imaging Features of Toxicities by Immune Checkpoint Inhibitors in Cancer Therapy. Curr Radiol Rep 2016;5:59.

25. Naidoo J, Wang X, Woo KM, et al. Pneumonitis in Patients Treated With Anti-Programmed Death-1/ Programmed Death Ligand 1 Therapy. J Clin Oncol 2017;35:709-17.

26. Shu CA, Gainor JF, Awad MM, et al. Neoadjuvant atezolizumab and chemotherapy in patients with resectable non-small-cell lung cancer: an open-label, multicentre, single-arm, phase 2 trial. Lancet Oncol 2020;21:786-95.

Cite this article as: Zhao H, Ning J, Gu Y, Zhang X, Yu W, Chen T, Luo Q. Consecutive severe immune-related adverse events after PD-1 inhibitor induction and surgery in locally advanced non-small cell lung cancer: a case report. Transl Lung Cancer Res 2021;10(8):3682-3688. doi: 10.21037/tlcr-21-603 